Editorial

\title{
Acknowledgement to Reviewers of Administrative Sciences in 2017
}

\author{
Administrative Sciences Editorial Office \\ MDPI AG, St. Alban-Anlage 66, 4052 Basel, Switzerland \\ Published: 10 January 2018
}

Peer review is an essential part in the publication process, ensuring that Administrative Sciences maintains high quality standards for its published papers. In 2017, a total of 37 papers were published in the journal. Thanks to the cooperation of our reviewers, the median time to first decision was 59.92 days and the median time to publication was 70 days. The editors would like to express their sincere gratitude to the following reviewers for their time and dedication in 2017:

\begin{tabular}{|c|c|c|}
\hline Alcaraz-Quiles, Francisco José & Highhouse, Scott & Park, Sangchan \\
\hline Aleksić, Ana & Hollensbe, Elaine & Patterson, Kathleen \\
\hline Alsaaty, Falih & $\mathrm{Hu}$, Kejia & Pereira, Dolores \\
\hline Amery, Fran & Hu, Shu-Kung & Perera, Luckmika \\
\hline Andrews, Rhys & Irving, Justin & Pircher Verdorfer, Armin \\
\hline Anonson, June & Irving, Justin & Plummer, Virginia \\
\hline Antunes, Pedro & Janousek, Christian & Psychogios, Alexandros \\
\hline Arkoubi, Khadija Al & Janousek, Christian & Purdy, Nancy \\
\hline Bariviera, Aurelio F. & Jaskyte, Kristina & Purdy, Nancy \\
\hline Baumann, Andrea & Jha, Chandan K. & Purdy, Nancy \\
\hline Belias, Dimitrios & Jones, Karen & Ragusett, Jared \\
\hline Bellman, Beryl & Junjan, Veronica & Ran, Bing \\
\hline Belso-Martínez, José Antonio & Kang, Miliann & Rekveldt, Marian Bosch \\
\hline Berry, Lois & Kimmerle, Joachim & Remke, Dr. Robyn \\
\hline Bockarie, Abu & Kisilowski, Maciej & Remke, Dr. Robyn \\
\hline Bockarie, Abu & Kluemper, Don & Roy, Michael J \\
\hline Bokhari, Hasnain & Kotilainen, Sirkku & Ryan, Susan \\
\hline Bolzani, Daniela & Kricheli-Katz, Tamar & Sadler-Smith, Eugene \\
\hline Bowles, Hannah Riley & Krueger, Joachim & Salas, Jesus M. \\
\hline Boyer, Teresa & Krueger, Joachim & Sanga, Kabini \\
\hline Bramley, Glen & Kumaran, Muthusami & Selck, Torsten J. \\
\hline Brett, Jeanne & Kumaran, Muthusami & Sermeus, Walter \\
\hline Browne, Liz & Kurucz, Elizabeth C. & Serpa, Sandro \\
\hline Bryant, Phillip & Lacroix, Martin & Siemienska, Renata \\
\hline Bygrave, Constance E. & Lai, Gavin & Singh, Mina \\
\hline Caetano, António & Landry, Anaïs Thibault & Sloan, Margaret \\
\hline Campbell, David & Lasson, Elliot & Sohmen, Victor \\
\hline Campbell, David & Laub, Jim & Sohmen, Victor \\
\hline
\end{tabular}




Cerchione, Roberto
Chen, Ziguang
Clarke, Sean P.
Cornelius, Nelarine
Corvo, Luigi
Dörfler, Viktor
Duchscher, Judy Boychuk
Eddy, Pamela L.
Ehrenhard, Michel
Eisler, Karen
Eley, Rob
Englmaier, Florian
Entwistle, Tom
Finegan, Joan
Fisher, Virginia
FitzGerald, Gerry
Fletcher, Luke
Flynn, Anthony
Forbes, Sarah
Fu, Chu-Chun
Fuller, Kay
Gallo, Maria
Gallo, Maria
Gamble, Brenda
Go Jefferies, Josephine
Grandy, Gina
Gunnarsdottir, Sigrun
Gunnarsdottir, Sigrun
Hannibal, Claire
Hartner-Tiefenthaler, Martina
Henseler, Jörg

\begin{tabular}{l} 
Laumer, Sven \\
Leavitt, Lynda \\
Lee, Allan \\
Lee, Mikyoung \\
Levasseur, Karine \\
Liou, James J. H. \\
Lisa, Carbone \\
Lofgren, Karl \\
Loftin, Collette \\
Loftin, Collette \\
MacPhee, Maura \\
Madsen, Susan R \\
Madsen, Susan R \\
Marcy, Richard \\
Marle, Franck \\
Martin, Jennifer \\
Mayers, Raymond Sanchez \\
Mayers, Raymond Sanchez \\
Mcpake, Barbara \\
Mease, Jennifer \\
Minerbi, Luciano \\
Molina-Morales, Francesc Xavier \\
Moorosi, Pontso \\
Mothersell, William \\
Nair, Girija \\
Narooz, Rose \\
Nguyen, Tam \\
Nicholas, John \\
Noordegraaf, Mirko \\
Olafsen, Anja Hagen \\
Ongaro, Edoardo \\
\hline
\end{tabular}

Sousa, Carlos M.P.

Spicer, David

Stephenson, Max O.

Stump, Erika

Talat, Usman

Tomazevic, Nina

Tseng, Ming-Lang

Tundys, Blanka

Tzanakou, Charoula

Udod, Sonia

Urzi Brancati, Cesira

Van Der Steen, Martijn

Van Der Steen, Martijn

Van Dierendonck, Dirk

Venkatraman, Sitalakshmi

Venkatraman, Sitalakshmi

Wagner, Joan

Warren, Clive

Warren, Lorraine

Warren, Lorraine

Wells, Paul

Wharton, Amy

White, Kate

Wierenga, Berend

Wollbrant, Conny

Wong, Vincent

Wong, Vincent

Woodhouse, Joan

Yao, YuChun

Zhao, Xiaohui

(C) 2018 by the authors. Licensee MDPI, Basel, Switzerland. This article is an open access article distributed under the terms and conditions of the Creative Commons Attribution (CC BY) license (http://creativecommons.org/licenses/by/4.0/). 